\title{
Regulatory roles of Oct proteins in the mammary gland
}

Xi Qian ${ }^{\mathrm{a}, \mathrm{b}}$, Feng-Qi Zhao ${ }^{\mathrm{a}, *}$

aLaboratory of Lactation and Metabolic Physiology, Department of Animal and Veterinary Sciences, University of Vermont, Burlington, Vermont 05405

${ }^{\mathrm{b}}$ Current address: Department of Pathology and Laboratory Medicine, University of Vermont, Burlington, Vermont 05405

* Corresponding author.

Email address: fzhao@uvm.edu

Short title: Oct proteins in the mammary gland

Abbreviations: C/EBP, CCAAT/enhancer-binding protein; GR, glucocorticoid receptor; GRE, glucocorticoid response element; MaSC, normal mammary stem cell; Oct, octamer-binding factor; Runx2, runt-related transcription factor 2; STAT5, signal transducer and activator of transcription 5 ; YY1, yin yang 1

\section{ABSTRACT}

The expression of Oct- 1 and -2 and their binding to the octamer motif in the mammary gland are developmentally and hormonally regulated, consistent with the expression of milk proteins. Both of these transcription factors constitutively bind to the proximal promoter of the milk protein gene $\beta$-casein and might be involved in the inhibition or activation of promoter activity via interactions with other transcription factors or cofactors at different developmental stages. In particular, the lactogenic hormone prolactin and glucocorticoids induce Oct-1 and Oct-2 binding and interaction with both the signal transducer and activator of transcription 5 (STAT5) and the glucocorticoid receptor on the $\beta$-casein promoter to activate $\beta$-casein expression. In addition, 
increasing evidence has shown the involvement of another Oct factor, Oct-3/4, in mammary tumorigenesis, making Oct-3/4 an emerging prognostic marker of breast cancer and a molecular target for the gene-directed therapeutic intervention, prevention and treatment of breast cancer.

Keywords: Breast cancer, Mammary gland, Milk protein, Oct protein, Transcriptional regulation

\section{Introduction}

In the human genome, approximately $10 \%$ of genes code for transcription factors, giving rise to $\sim 2,600$ transcription factors in the proteome [1]. Transcription factors are one of the families of proteins that read and translate the genetic landscape of the DNA. They recognize and bind to sequence-specific cis-acting DNA elements located within regulatory regions, such as enhancers and promoters, of the genes that they regulate. Depending on the transcription factors, the transcription of the regulated genes is either up- or down-regulated [2]. As such, transcription factors play crucial roles in many essential cellular processes.

The octamer-binding transcription factors (Octs) were originally defined by their ability to bind to an 8-bp consensus DNA sequence [ATT(T/A)GCAT] (referred to as an 'octamer motif') and closely related sequence variants [3, 4]. Electrophoretic mobility shift assays (EMSAs) of nuclear extracts purified from mouse cell lines and tissues at different developmental stages with an octamer motif-containing DNA probe revealed 11 distinct band shifts, indicating the existence of 11 Oct factors (Oct-1 to -11) [3, 5, 6]. However, only eight Oct factors thus far, Oct-1 (POU2F1) [7], Oct-2 (POU2F2) [8], Oct3/4 (POU5F1) [9], Oct-6 (POU3F1) [10], Oct-7 (POU3F2) [11, 12], Oct-8 (POU3F3) [13], Oct-9 (POU3F4) [14], and Oct-11 (POU2F3) [5], have been cloned and characterized. At least some of the remaining band might represent degraded products or the splicing variants of these genes. Structural analysis of the Oct factors revealed that they constitute a subclass of the POU factor family, of which the founding members are Pit-1, Oct-1/2, UNC-86 [15]. POU factors themselves belong to a much larger homeodomain protein family. Oct factors can positively or negatively regulate a wide variety of ubiquitously expressed or tissue-specific genes [16]. For example, Oct-1 transactivates housekeeping genes, such as histone $\mathrm{H} 2 \mathrm{~b}$ [17] and TBP-associated factor 12 [18], as well as mammary gland-specific milk protein genes $[19,20]$. In contrast, it downregulates the expression of various genes, including vascular cytochrome P450 (CYP) monooxygenases [21], homeodomain transcription factor Cdx2 [22], and vascular cell adhesion molecule (VCAM-1) [23]. As such, Oct factors have been implicated in many physiological and pathophysiological processes, such as embryogenesis [24], stress responses [25], lactation [26], carcinogenesis [27], and diabetes [28].

The mammary gland (breast in humans), which distinguishes mammals from all other animals, is a reproductive organ that provides neonatal offspring with milk for 
nourishment and disease resistance. The mammary glands of dairy animals, such as cows and goats, also produce abundant milk that represents an important nutrient source in modern human life and a multi-billion dollar industry. As a unique organ, the mammary gland undergoes its development predominantly after birth. To achieve full functional differentiation (i.e., copious milk synthesis and secretion), the mammary gland undergoes a dramatic series of changes in morphology, size, composition, and activity throughout the life cycle of mammals [29]. Unfortunately, the mammary gland is susceptible to cancer that takes away millions of people's lives each year. In contrast to breast tumors and the normal mammary stem cell (MaSC) population that express Oct1, Oct-2, Oct-3/4, and Oct-11 [30, 31], normal differentiated mammary tissue predominantly expresses Oct-1 and Oct-2 [20, 30, 32]. This review is aimed at summarizing the known and potential roles of Oct proteins in regulating mammary gland development, lactation, and breast tumorigenesis.

\section{An overview of Oct transcriptional factors}

Homology comparisons of the DNA-binding domains of POU family members define six distinct POU protein subclasses, named POU1 through POU6 [15]. Further phylogenetic analyses of all known Octs also suggest that known Oct proteins comprise POU domain transcription factor classes II (Oct-1, -2, and -11), III (Oct-6, -7, -8, and -9), and V (Oct-3/4) [16, 33]. Notably, only POU II, III, and V, but not the other three subclasses, display high affinity for the canonical octamer sequence [16]. All known Oct factors, such as other POU family members, share a characteristic bipartite DNAbinding POU domain, which is composed of the POU-specific subdomain $\left(\mathrm{POU}_{\mathrm{S}}, \sim 80\right.$ aa) and the POU-homeodomain $\left(\mathrm{POU}_{\mathrm{H}}, \sim 60 \mathrm{aa}\right)$ that are tethered by a flexible linker (14-26 aa) [34]. The two subdomains make corresponding contacts with the octamer motif. In particular, the $\mathrm{POU}_{\mathrm{S}}$ associates with the 5'-half site of the octamer motif, ATGC, whereas the $\mathrm{POU}_{H}$ partners with 3'-half site [35]. High-affinity and site-specific DNA binding depends on the cooperation of $\mathrm{POU}_{S}$ and $\mathrm{POU}_{\mathrm{H}}$. These two subdomains are independently folded and bind to the opposite faces of the DNA within two adjacent major grooves via a helix-turn-helix $(\mathrm{HTH})$ structure [36, 37]. In addition, Oct factors can also bind to octamer-derived variant sequences as dimers or multimers [38,39]. For instance, the Oct-1 homodimer binds to the more octamer-related palindromic element (MORE) sequence that is a combination of paired and inverted octamer half-sites [40]. The structural characteristics and DNA-binding properties of Oct factors have been well reviewed elsewhere [16, 29].

It is generally believed that except for the universal expression patterns of Oct-1, all other Oct factors exhibit highly tissue-restricted expression patterns in adults [16, 33]. However, another POU II-subclass member, Oct-2, originally considered to be expressed only in the brain, lymphocytes, and blood, is found to also be expressed in many other tissues including the mammary gland [32]. Oct-1 and Oct-2 share $\sim 88 \%$ similarity in the POU domain cDNA sequences and have nearly identical DNA-binding specificities [41]. Correspondingly, Oct-1 and Oct-2 share functional properties. For example, both transcription factors regulate immunoglobulin gene expression [42, 43] and exhibit interdependent roles in mouse development [44]. The last known POU II Oct factor is Oct-11, which is primarily expressed in the epidermis and plays an important 
role in epidermal keratinocyte differentiation $[45,46]$. POU III Oct factors include Oct-6, $7,-8$, and -9 ; interestingly, all of the genes encoding these factors lack introns [16]. Oct factors of this class are predominantly expressed within the central nervous system and play both overlapping and specific roles in the development and functions of the nervous system [16]. For example, although Oct-8 can fully replace the role of Oct- 6 in regulating Schwann cell development and myelination [47], replacing Oct-6 with Oct-8 in mice results in severe defects in forebrain development $[47,48]$. Oct-3/4, the only known POU V Oct factor, is only expressed in totipotent mouse and human embryonic stem and germ cells [33]. In these cells, Oct-3/4 regulates and maintains cell pluripotency and self-renewal. Notably, the expression of Oct-3/4 is down-regulated in differentiating embryonic stem cells, and its expression disappears in the normal somatic cells of differentiated tissues $[49,50]$. Oct- $3 / 4$ therefore tends to be widely expressed during early developmental stages and gradually becomes restrictively expressed as development proceeds.

Important biological processes are commonly regulated and controlled at multiple levels. This is also true for transcription factors. Not only do transcription factors control the rates of transcription to regulate the amounts of gene products available within the cell, but they themselves are regulated by environmental stimuli, which modulate their expression levels, activities, subcellular localization, and association with other transcription factors or co-factors. Such regulations of Oct factors have recently been well reviewed elsewhere [51]. Notably, by associating with chromatin modifying factors, Oct factors are able to switch between repressive and activating modes, as evidenced by the example of Oct-1 regulation of Polr2a and Ahcy gene expression in murine fibroblasts and of the IL-2 and Cdx-targeted locus in T lymphocytes and cancer cells [52]. Consistent with this finding, Oct-3/4 has recently been shown to function via a similar mechanism in the regulation of cell pluripotency [53]. This property might lead a single transcription factor to regulate gene transcription at multiple levels in complex developmental processes.

\section{Expression and binding activity of Oct factors in the mammary gland}

The expression of Oct-1 and Oct-2 in normal mouse mammary gland has been demonstrated by qRT-PCR, Western blotting, and gel-shift assays $[4,20,32,54,55]$. Further immunocytochemical analysis has revealed that Oct-1 and Oct-2 are expressed in the alveolar epithelial cells of mouse mammary gland with primary subcellular localization to the nucleus $[32,55,56]$. The expression of both Oct-1 and Oct-2 are developmentally regulated in the mouse mammary gland (Table 1). In particular, the expression of Oct-1 mRNA was shown to be increased in the primary mammary epithelial cells from virgin to early, mid-, and late pregnancy-stage mice, peaking in expression during the lactation stage [57]. Oct-2 protein levels in mammary nuclear extracts exhibit a trend similar to that of Oct-1 mRNA [32]. The expression patterns of both Oct-1 and Oct-2 coincide with that of milk proteins [58], the expression of which is well-known to be induced by lactogenic hormones during late-pregnancy and lactation, suggesting that lactogenic hormones are also responsible for observed increases in the expression of Oct-1 and Oct-2. However, the lactogenic hormonal stimulation of HC11 cells, a murine mammary epithelial cell line isolated from mid-pregnant mice that retains 
the responsiveness to lactogenic hormones [59], results in a significant increase in the expression of the milk protein $\beta$-casein without affecting the mRNA and protein expression levels of Oct-1 and Oct-2 [54, 55]. Thus, the expression of Oct-1 and Oct-2 within the mammary gland might be regulated by factors other than lactogenic hormones including other hormones, local growth factors, and extracellular matrix, the concerted actions of which are indispensable for mammary gland development and functional differentiation [26]. In addition to Oct-1 and Oct-2, another Oct factor, Oct-3/4, has also been found to be expressed in the normal mammary stem cells (MaSCs) (see below) $[31,60]$.

Nuclear extracts from mouse mammary glands form at least two binding complexes (DS1 and DS2) on the DNA oligonucleotide sequence corresponding to the octamer motif $[19,20,55]$. It appears that both the DS1 and DS2 complexes contain both Oct-1 and Oct-2 proteins, as both complexes could be either supershifted by Oct-1 antibody or blocked by Oct-2 antibody [55]. However, what causes the size differences between the two complexes remains unknown. The maximal increase in binding activity of DS1 and DS2 in the mammary gland occurs at late pregnancy (Table 1) [19]. In mammary gland explant cultures, the DS1 activity is increased by epidermal growth factor or a combination of prolactin and hydrocortisone $[19,20]$. In addition, the in vivo treatment of virgin mice with progesterone or a combination of estradiol and progesterone induces DS1 activities in the mammary gland [20].

\section{Functional roles of Oct factors in the mammary gland}

The developmental and hormonal regulation of Oct-binding activity in the mammary gland suggests that Oct factors play regulatory roles in this tissue. Indeed, studies have shown that Oct factors are involved in the regulation of milk gene expression and possibly in mammary development.

\subsection{Regulation of milk protein genes}

Sequence alignments of the proximal promoters of milk casein genes from a wide range of species reveal a highly conserved octamer motif adjacent to the transcription start site (Fig. 1A) [19], indicative of a potential functional role of Oct factors in regulating milk gene expression. Studies on the regulation of milk protein genes by Oct factors have generally focused on the $\beta$-casein gene. $\beta$-casein is a major milk protein synthesized in the mammary gland during lactation and is regarded as a marker for the functional differentiation of mammary epithelial cells. Furthermore, the $\beta$ casein gene promoter has been studied for decades as a model for exploring the mechanisms by which lactogenic hormones (prolactin and glucocorticoids) synergistically regulate gene expression [26]. Sequence alignment of the'5flanking regions of the human, rat, cow, sheep, and goat $\beta$-casein genes has revealed two evolutionarily conserved regions, the proximal promoter and the distal enhancer (Fig. $1 A \& B)[61,62]$. These two regions contain composite binding sites for different transcription factors, including the signal transducer and activator of transcription 5 (STAT5), glucocorticoid receptor (GR), CAAT/enhancer binding protein (C/EBP) $\beta$, and yin yang 1 (YY-1) $[26,63]$. The importance of these transcription factors in regulating $\beta$ - 
casein gene expression has been established by studies using transient transfection experiments and transgenic and knockout mouse models [63]. Because none of these transcription factors is mammary specific, mammary-specific $\beta$-casein gene expression is believed to be dependent on the combinatorial protein-protein interactions among these transcription factors and co-factors at the composite regulatory elements present in both the proximal promoter and the enhancer in response to lactogenic hormones. Moreover, recent studies using a chromosome conformation capture assay demonstrated that these two regulatory regions interact with one another via chromatin looping as follows: lactogenic hormones induce the physical interaction between the $\beta$ casein gene proximal promoter and the distal enhancer [64] and that withdrawal of lactogenic hormones results in the disruption of this interaction, accompanied by a decrease in $\beta$-casein gene expression [65].

Based on the previously published results, a working model has been proposed to elucidate how the signaling pathways regulated by lactogenic hormones are integrated to activate $\beta$-casein expression [66]. In this model (Fig. 2), YY-1 binds to the proximal promoter of the $\beta$-casein gene in the absence of lactogenic hormones and forms a repressive complex by recruiting histone deacetylase 3 (HDAC3) and LIP (inhibitory isoform of C/EBP 3 ) [67-69], promoting a transcriptionally repressive chromatin organization in association with deacetylated histone $\mathrm{H} 3$ [70] and dimethylated lysine 9 of histone $\mathrm{H} 3$ (H3K9) [71]. Deacetylated $\mathrm{H} 3$ is potentially promoted by HDAC3 [64], although the precise mechanism by which dimethylated $\mathrm{H} 3 \mathrm{~K} 9$ is maintained is unknown. After stimulation with lactogenic hormones, the transcription factors STAT5, GR, and LAP (activating isoform of C/EBP $\beta$ ) rapidly bind to their respective binding sites within the $\beta$-casein gene proximal promoter and distal enhancer [70], displace the repressive complex, and subsequently recruit the nuclear co-activator p300 via protein-protein interactions [72]. The recruited coactivator p300 facilitates histone $\mathrm{H} 3$ acetylation, relaxing the chromatin structure at the $\beta$-casein cisregulatory regions via its intrinsic histone acetyltransferase (HAT) activity. Although glucocorticoids alone trigger a rapid increase in histone $\mathrm{H} 3$ acetylation, treatment with prolactin and glucocorticoids in combination is required for the stable association of p300 at both the proximal promoter and the distal enhancer regions of the $\beta$-casein gene [65]. Lactogenic hormones also promote open chromatin structure by inducing H3K9 demethylation via undiscovered mechanisms. Interactions among these activated transcription factors and co-activators result in physical contact between the proximal promoter and the distal enhancer via DNA looping, which facilitates the recruitment of the preinitiation complex and $\beta$-casein transcription [64].

Studies in our laboratory and others have shown that Oct-1 and Oct-2 also play a key role in the regulation of $\beta$-casein transcription described above. We previously discovered that Oct-1 and Oct- 2 constitutively bind to and induce the basal activity of $\beta$ casein gene promoter in the absence of lactogenic hormones [4, 20, 56]. However, lactogenic hormones further increase their binding to the promoter [54, 55]. Our transfection experiments indicated that Oct-1 and Oct- 2 function as transactivators in lactogenic hormones-stimulated $\beta$-casein gene expression in mammary epithelial cells $[54,55]$. Oct-1 has recently been shown to interact with and to facilitate the binding of the Runt-related transcription factor 2 (Runx2), the binding site of which is adjacent to the octamer motif, at the $\beta$-casein gene promoter (Fig. 1A) [73]. The co-overexpression 
of Oct-1 and Runx2 results in increased basal $\beta$-casein gene promoter activity compared to that of the overexpression of either protein alone [73]. In addition, we demonstrated that lactogenic hormones induce physical interactions of either Oct-1 or Oct-2 or both with both STAT5 and GR and increase $\beta$-casein gene promoter activity $[54,55]$. Oct-1 facilitates the binding of both STAT5 and GR at the $\beta$-casein gene promoter, similar to the effect of Oct-1 on Runx2 binding [54]. Furthermore, progesterone is a reproductive hormone that inhibits $\beta$-casein gene expression by inducing the binding of the progesterone receptor (PR) at the $\beta$-casein promoter $[71,74]$. However, it also induces Oct-1-binding activity at the $\beta$-casein gene promoter in the mammary gland [20]. Because Oct-1 can interact with the PR [75], it might also play an inhibitory role in $\beta$-casein gene expression by interacting with the PR. Collectively, we expand on Rijnkels's model [66] described above to propose a working model of regulating $\beta$-casein gene expression involving Oct-1 and Oct-2 (Fig. 2). Depending on upstream signaling, Oct-1 and Oct-2 can act as either transactivators or repressors to switch $\beta$-casein gene expression between the inducible or repressive states in mammary epithelial cells, as follows. 1) In the quiescent state, Oct-1, and potentially Oct-2, promotes an inhibitory state for the $\beta$-casein gene by interacting with YY1, LIP, and HDAC3. 2) Following progesterone stimulation, the PR is recruited and stabilizes the repressive complex, further inhibiting $\beta$-casein gene expression. 3) Following lactogenic hormone stimulation, however, Oct-1 and Oct-2 switch the $\beta$-casein gene to a permissive state by interacting with phosphorylated STAT5, GR, LAP, and Runx2, promoting pre-initiation complex formation and inducing $\beta$-casein gene expression.

Supporting this hypothesis, previous studies, as mentioned above, have also shown that Oct-1 and other Oct proteins can serve as switchable regulators in controlling their target gene expression. Depending on upstream signaling, the Octtargeted genes can either be rapidly induced or become stably repressed. One key example is the regulation of the interleukin 2 gene by Oct-1 in CD4 T lymphocytes [52]. In naïve T lymphocytes, Oct-1 recruits the Mi-2/NuRD chromatin-remodeling complex to the interleukin 2 gene, inducing extensive methylation at the CpG site proximal to the transcription start site, resulting in repression of gene expression. In contrast, upon T lymphocyte activation, Oct-1 loses its ability to associate with Mi-2/NuRD, instead indirectly or directly recruiting the Jmjd1a/KDM3A histone demethylase to the interleukin 2 gene, blocking gene repression by opposing inhibitory histone methylation [52]. Oct-1 might elicit a similar function in regulating the MMTV promoter [76] and the Polr2a, Ahcy, and Cdx2 genes [52]. Oct-4, another member of the Oct family, also has exhibited a bipotential function in regulating target genes involved in embryonic stem cell (ESC) development, such as Hoxa5, Otx2, Pou4f1, Hoxc6, and Pax6 [77, 78]. Further investigation is required to verify this bi-potential or switchable role of Oct-1 and Oct-2 in $\beta$-casein gene regulation in response to hormonal treatments. Specifically, the presence of Oct-interacting co-factors and the chromatin status of the $\beta$-casein gene promoter must be investigated under different hormonal treatments. One possibility is that, as shown in our model in Fig. 2, Oct-1 and Oct-2 recruit the histone-lysine Nmethyltransferase SETDB1, which was found to be associated with Oct-4 $[79,80]$, to promote the formation of dimethylated $\mathrm{H} 3 \mathrm{~K} 9$ at the $\beta$-casein proximal promoter in the absence of lactogenic hormones [71]. However, after lactogenic hormone treatment, 
Oct-1 and Oct-2 might recruit Jmjd1a/KDM3A histone demethylase to the $\beta$-casein gene, removing inhibitory histone methylation.

How Oct proteins respond to upstream signals and hormones and how they interact with other proteins to regulate target gene transcription remains unknown. Previous studies have reported that Oct proteins can integrate various upstream signals that precisely regulate Oct proteins at multiple levels, including at the levels of expression, localization, and activity [51]. Most Oct proteins are subject to posttranscriptional modifications, including phosphorylation, O-GIcNAcylation, SUMOylation, and ubiquitylation [16]. These post-transcriptional modifications are likely responsible for many changes in Oct proteins in terms of stability, localization, and activity [51]. We have previously shown that the binding activities of both Oct- 1 and Oct- 2 to the $\beta$-casein promoter can be induced by lactogenic hormones and that this induction is not mediated by either increased expression or nuclear translocation [26, 54, 55]. Therefore, lactogenic hormones likely enhance Oct-1 and Oct-2 DNA-binding activity via mechanisms of post-translational modification or protein-protein interactions. The posttranslational modifications of Oct proteins, particularly phosphorylation, might mediate the assembly of multiprotein transcriptional regulatory complexes [81, 82].

The results from our previous study indicate that Oct-2 interacts with Oct-1 regardless of stimulation with lactogenic hormones. However, the interaction between Oct-1 and Oct-2 does not synergistically stimulate $\beta$-casein gene expression in response to lactogenic hormones [55]. Oct-1 and Oct-2 appear to be functionally redundant in the hormonal regulation of the $\beta$-casein gene. This observation is consistent with previous reports of Oct-1 and Oct-2 knockout mice in which Oct-1 and Oct-2 operate redundantly in regulating B cell development and IgG transcription [83]. These data also imply that Oct- 1 and Oct- 2 form heteromers at the $\beta$-casein gene promoter. This possibility is supported by the fact that Oct proteins interact not only with various transcription factors and co-activators [33] but also with themselves to form homo-/hetero-dimers or even higher-order complexes [38, 40, 84].

\subsection{Regulation of mammary gland development}

Mammary gland development is a finely regulated process. Although it begins during embryonic development, it occurs largely postnatally under the control of female reproductive hormones estrogen, progesterone, and prolactin. During the major postnatal developmental stages, pubertal growth, pregnancy, lactation, and involution, the mammary gland is subject to fundamental morphological and functional changes. These different stages are correspondingly associated with a series of mammary epithelial cell proliferation, differentiation, and apoptotic events [85]. Each of these processes is dependent on the activity of specific sets of transcription factors that determines coordinated changes in the gene expression profiles of the cells [85].

Oct-1 and Oct-2 are implicated in mammary development, as their binding activity is developmentally regulated in the mammary gland, as follows: low in virgin and involution stages and high in pregnancy and lactation stages [19]. However, the effects of knockout of these factors on mammary development have not, to our knowledge, been studied. However, a limited numbers of studies have suggested a possible role for Oct-1 in the mammary involution process $[86,87]$. Involution occurs at the weaning 
stage, when the mammary gland undergoes a reductive remodeling process associated with cell apoptosis and the cessation of milk protein gene expression. The high Oct-1 DNA-binding activity in mammary gland extracts at lactation is almost undetectable at the $4^{\text {th }}$ day of involution [88]. This modulation was further substantiated at the protein level, either by protein degradation or post-translational modification, as Oct-1 mRNA level is unchanged at the $4^{\text {th }}$ day post weaning [88]. Oct-1 is crucial for the expression of several housekeeping genes including histones and small nuclear RNAs, and the transcription of these genes is turned off during involution [88]. Therefore, the loss of the DNA-binding activity of Oct-1 might be incompatible with cell survival. The activity of protein kinase A (PKA) in the mammary gland protein extracts has been reported to be dramatically increased within hours after weaning, and recombinant PKA protein suppresses Oct-1 DNA-binding activity [88, 89], suggesting that the loss of Oct-1 DNAbinding activity during mammary involution is a direct consequence of PKA-mediated phosphorylation. This possibility is supported by a recent study in which the activation of PKA by increasing CAMP levels was found to be correlated with the induction of apoptosis in the malignant glioma cell line A-172 [90]. Nonetheless, the precise role of Oct-1 in mammary involution, the involvement of PKA activation, and the upstream signaling pathways and downstream targets of Oct-1 require further investigation.

Oct-3/4, together with SOX2 and NANOG, is essential for the maintenance of pluripotency and survival of embryonic stem cells (ESCs). The re-expression of Oct-3/4 and three other embryonic transcription factors is sufficient to reprogram adult somatic cells into an undifferentiated, pluripotent, and self-renewable state (referred to as induced pluripotent stem cells; iPSCs) [91]. In addition to ESCs and iPSCs, it has become increasingly recognized that a subpopulation of cells in various adult organs, including the mammary gland, also expresses Oct-3/4 and other embryonic transcription factors [31, 60, 92]. In the mammary gland, this subpopulation of cells is called MaSCs, which are primarily localized within the epithelium, with small populations within the stroma [31, 60]. Imbalanced up-regulation of Oct-3/4 expression in MaSCs or the aberrant expression of Oct-3/4 in other cells might lead to the generation of cancer stem cells (CSCs) and subsequent malignant transformation (see below).

\section{OCT factors in breast tumorigenesis}

An increasing body of evidence supports the potential functions of Oct factors, particularly Oct-3/4, in breast tumorigenesis. Oct-3/4 has been detected in a few types of somatic tumors, including breast cancer. Reactivation of Oct-3/4 expression has been observed in several malignant human breast cancer cell lines, including MCF-7, SKBr3 and MDA-MB-453, but not in normal human breast cells $[30,50,93]$. Immunofluorescence staining has revealed heterogeneous expression of Oct-3/4 in MCF-7 cells [50], as well as in breast cancer cells in tissue sections [94]. In breast cancer cells, Oct-3/4 expression is repressed by all-trans-retinoic acids and is correlated with decreased cell proliferation [93]. The expression of Oct-3/4 in these cells upregulates the expression of fibroblast growth factor-4 (FGF-4) [93], a gene which stimulates MCF-7 cells to become more tumorigenic and metastatic in ovariectomized and tamoxifen-treated nude mice [95]. Furthermore, the overexpression of Oct-3/4 and NANOG in breast CSCs enhances their invasiveness, whereas the knockdown of these 
two factors inhibits their migration in vitro [96]. These observations indicated a link between the expression levels of Oct-3/4 and breast cancer cell tumorigenicity.

To examine Oct4 expression in primary breast carcinomas, we recently obtained 28 primary breast tumor samples from patients following lumpectomy or mastectomy and 9 normal breast tissues from women undergoing cosmetic surgery for plastic implantation. Quantitative RT-PCR analysis revealed that all tumor tissues irrespective of their types (adenocarcinoma, fibroadenoma or ductal in-situ carcinoma) expressed Oct-3/4 in their transcriptome at levels that were several thousand-fold increased compared to normal breast tissues. In addition, Oct-3/4 protein was detected in all 4 tumor but not normal breast tissues (Misra and Zhao, unpublished observation). Consistent with our observations, the expression of Oct-3/4 was found to be significantly correlated with poor prognosis in 126 breast cancer patients [96]. Thus, Oct3/4 represents a potential prognostic marker for breast cancer.

The expression of Oct-3/4 in breast cancer supports the hypothesis that adult stem cells serve as the target cell for carcinogenesis [97, 98]. Oct-3/4-expressing breast CSCs isolated from breast cancer lesions exhibit stem/progenitor cell properties [99]. Whether the expression of Oct-3/4 is required for the maintenance of the transformed state of cancer cells remains an important open question. If the answer is yes, then Oct$3 / 4$ represents an excellent target for gene-directed therapeutic intervention for the prevention and treatment of breast cancer.

Notably, computational analysis of the human genome has identified six putative Oct-3/4 pseudogenes [100]. The transcription of these pseudogenes was found in cancer cells and primary tumors [101]. Thus, it is possible that the expression of Oct-3/4 pseudogene mRNAs contribute artifacts in previous reports of Oct-3/4 expression in cancers. In addition, two alternatively spliced Oct-3/4 isoforms, Oct3A and Oct3B, have been cloned [102]. To verify the expression of the Oct-3/4 gene and to identify the Oct$3 / 4$ isoforms expressed in breast cancer cells, our laboratory performed Rapid Amplification of CDNA Ends (RACE) in MCF-7 and in the non-malignant breast cell line, MCF-10A. RACE analysis and subsequent full-length cDNA cloning revealed that MCF7 cells indeed express multiple alternatively spliced isoforms of the Oct- $3 / 4$ gene on chromosome 8 (we proposed the name Oct-3 [16], Fig. 3), whereas MCF-10A cells express multiple alternatively spliced, untranslated transcripts of an Oct-3/4 gene present on chromosome 6 (we proposed the name Oct-4 [16], Fig. 3). These results were also verified in primary breast tumors and normal breast tissues (Misra and Zhao, unpublished observation). Thus, cancerous and normal breast cells might express different Oct-3/4 genes, which exhibit a 96\% sequence identity (Fig. 3).

The Oct-3/4 paralog Oct-1 might also be involved in the cancer stem cell phenotype in breast cancer, as evidenced by the finding that elevated Oct-1 protein expression correlates with increased $\mathrm{ALDH}{ }^{\mathrm{HI}}$ and $\mathrm{CD} 24^{\mathrm{LO}} \mathrm{CD} 44^{\mathrm{HI}}$ stem cell-like populations in tumor cell lines and primary breast cancer samples, respectively [103].

\section{Conclusions and perspectives}

Increasing evidence demonstrates that Oct factors play important roles in the regulation of mammary gland development, lactation, and tumorigenesis. Although the current evidence is compelling, it is just a preface; significantly more research is required to establish these functional roles and to study the underlying mechanisms. 
Specifically, the following key and urgent questions remain unanswered. Are other Oct factors also expressed that play roles in mammary gland biology at specific developmental stages? What are the phenotypic changes in mammary development and lactation when individual or a multiple Oct factors are overexpressed or knocked down in the mammary gland? What are the target genes that Oct factors regulate in the mammary gland at different developmental stages? How do lactogenic hormones increase Oct-1 and Oct-2 binding to the milk protein gene promoters? Finally, does the overexpression of Oct-3/4 in MaSCs or the aberrant expression of Oct-3/4 in somatic cells within the mammary gland induce mammary tumorigenesis? Furthermore, does the knockdown of Oct-3/4 in breast cancer cells result in tumor regression? Answers to these questions will provide mechanistic insights into mammary development and lactation, which will help to improve milk production in dairy animals, as well as provide targets for the diagnosis, prevention, and treatment of breast cancer in humans.

\section{Acknowledgements}

The author's work in this review was supported by the USDA Experimental Station Hatch Grant (VT-H01508), the Department of Defense Breast Cancer Research Program Concept Award (\#W81XWH-05-1-0432), and the Vermont Cancer Center VCC/LCCRO Pilot Award (\#022820). 
Table 1. Relative expression levels and DNA-binding activities of Oct-1 and -2 during the mammary gland developmental cycle.

\begin{tabular}{|c|c|c|c|c|c|c|}
\hline & \multirow{2}{*}{ Virgin } & \multicolumn{3}{|c|}{ Pregnancy } & \multirow{2}{*}{ Lactation } & Involution \\
\cline { 3 - 5 } & & early & mid & late & & n.d. \\
\hline $\begin{array}{c}\text { Oct DNA-binding } \\
\text { activity }\end{array}$ & n.d. & $\uparrow$ & $\uparrow \uparrow$ & $\uparrow \uparrow \uparrow$ & $\uparrow \uparrow$ & $\uparrow \uparrow \uparrow \uparrow$ \\
\hline $\begin{array}{c}\text { Oct-1 expression } \\
\text { level (mRNA) }\end{array}$ & $\uparrow$ & $\uparrow \uparrow$ & $\uparrow \uparrow \uparrow$ & $\uparrow \uparrow \uparrow$ & $\uparrow \uparrow \uparrow \uparrow$ & n.d. \\
\hline $\begin{array}{c}\text { Oct-2 expression } \\
\text { level (protein) }\end{array}$ & n.d. & $?$ & $\uparrow \uparrow \uparrow$ & $\uparrow \uparrow \uparrow \uparrow$ & $\uparrow \uparrow \uparrow$ & n.d \\
\hline
\end{tabular}

Note: Multiple $\uparrow$ indicates proportionately increased expression levels or DNA-binding affinity to octamer motif compared with other stages. n.d. indicates not detectable. Question marks indicate no corresponding information available. These data were summarized from previously published results $[19,32,57]$. 


\section{Figure legends}

Fig. 1. Schematic representation of the mouse $\beta$-casein gene regulatory regions. Transcription factor binding sites mapped in the proximal promoter $(A)$ and 5 ' distal enhancer $(B)$ of the $\beta$-casein gene. Signal transducer and activator of transcription 5 (STAT5), CCAAT/enhancer-binding protein (C/EBP), and yin yang 1 (YY-1)-binding sites and half glucocorticoid response elements ( $1 / 2$ GREs) for the glucocorticoid receptor (GR) in the $\beta$-casein proximal promoter and/or distal enhancer have been functionally verified and extensively studied [26, 63]. The binding sites for octamer factors (Oct) and runt-related transcription factor 2 (Runx2) have recently been characterized [26]. Sequences for the octamer and Runx elements in the promoters of $\alpha-S 1$ and $\beta$-caseins from several species are aligned and boxed. DNA-binding sites for transcription factors are shown in different shapes.

Fig. 2. A working model illustrating the hormonal regulation of $\beta$-casein gene transcription in mammary epithelial cells. This model is adapted from a model previously proposed by Rijnkels' et al. (2012) [66]. In the quiescent state, yin yang 1 (YY-1) binds to the $\beta$-casein proximal promoter and forms a repressive complex by recruiting histone deacetylase 3 (HDAC3) and liver-enriched transcriptional inhibitory protein (LIP, a dominant negative isoform of CAAT/enhancer-binding protein $\beta$, C/EBP $\beta$ ), resulting in the formation of a negative histone marker, deacetylated histone $\mathrm{H} 3$, at the proximal promoter of the $\beta$-casein gene [64]. Oct-1 and/or Oct-2 might bind to the octamer motif at the $\beta$-casein gene proximal promoter as a monomer or a high-order complex, such as a heterodimer or a homodimer. Oct(s) might recruit cofactors, including histone-lysine $\mathrm{N}$-methyltransferase SETDB1, leading to the formation of dimethylated lysine 9 of histone H3 (H3K9). Under progesterone (PG) stimulation, the progesterone receptor (PR) binds to the half glucocorticoid response element ( $1 / 2 \mathrm{GRE})$ at the $\beta$-casein gene promoter [74], which might further stabilize the repressive complex by enhancing Oct(s) binding. When the mammary epithelial cells are stimulated by prolactin (PRL) and glucocorticoids (GC), Oct(s) might be activated by post-translational modification and switch to function as a transactivator. Activated Oct- 1 might recruit its coactivator(s) to the $\beta$-casein gene, removing inhibitory histone methylation marks. PRL and GC result in rapidly binding of STAT5, glucocorticoid receptor (GR), and C/EBP $\beta$ (positive isoform) to their respective response elements within $\beta$-casein regulatory regions, recruiting p300 through protein-protein interactions and, in turn, facilitating histone acetylation [64]. Additionally, the interactions of Oct-1 with STAT5 and GR can stabilize the binding of STAT5 and GR to the $\beta$-casein promoter [54]. Interactions between the promoter and enhancer, which are mediated via these transcription factors and coactivators, enable DNA looping. The formation of the active chromatin loop between regulatory elements facilitates and stabilizes binding of the basal transcriptional machinery to the DNA template and initiates transcription. 
Fig. 3. Sequence alignment of the human Octamer-binding factors Oct-3 and Oct4A. The GenBank protein identification numbers of these sequences are NP_001153014.1 (Oct-3) and NP_002692.2 (Oct-4A). The alignment was performed using the CLUSTAL-W program with an open gap cost $=10$ and a gap extension cost $=$ 0.2 . Residues that are highlighted with black shading represent conserved amino acids, and the gray shading indicates non-conserved amino acids. The conserved amino acids are shown on the bottom of the sequence alignment. 
Fig. 1

A

\section{$\beta$-Casein gene proximal promoter}

$1 / 2$ GRE $\quad 1 / 2$ GRE $\quad 1 / 2$ GRE $\quad 1 / 2$ GRE $1 / 2$ GRE $1 / 2$ GRE

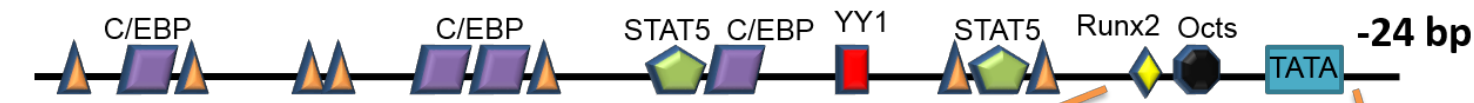

-232 bp

Mouse $\beta$-casein

Rat $\beta$-casein

Cow $\beta$-casein

Rabbit $\beta$-casein

Human $\beta$-casein

Yak $\beta$-casein

Goat $\beta$-casein

Rat $\alpha$-s1-casein

Cow $\alpha$-s1-casein

Rat $\alpha$-s2-casein-like A
TCTTACAA ACCACA AA ATTAGCATGTCATTAA TCTTACGA ACCACAA-ATTAGCATGTCATTAA TATTTCAA ACCACA AA ATTAGCATGCCATTAA TTTTTCAA ACCACA AA ATTAGCATGTCATTAA GAACTGAAACCACA AA ATTAGCATTTT-CTAA TATTTCAA ACCACA AA ATTAGCAT GCCATTAA TATTTCAAACCACA AA ATTAGCATGCCATTAA GAAACAAA ACCACA AA ATTAGCATTTCACTGC AACTG-AA ACCACA AA ATTAGCATTTTACTAA TATTTGAAACCACA GAATTAGCATATGATGCT

Runx Oct

B

$\beta$-Casein gene distal enhancer

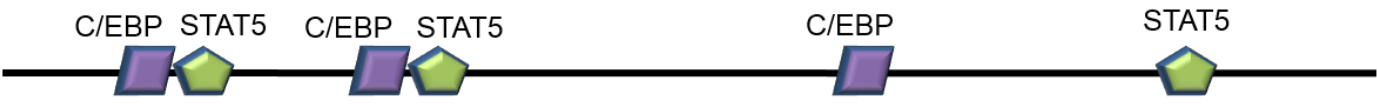

$-6.4 k b$ 
Fig. 2
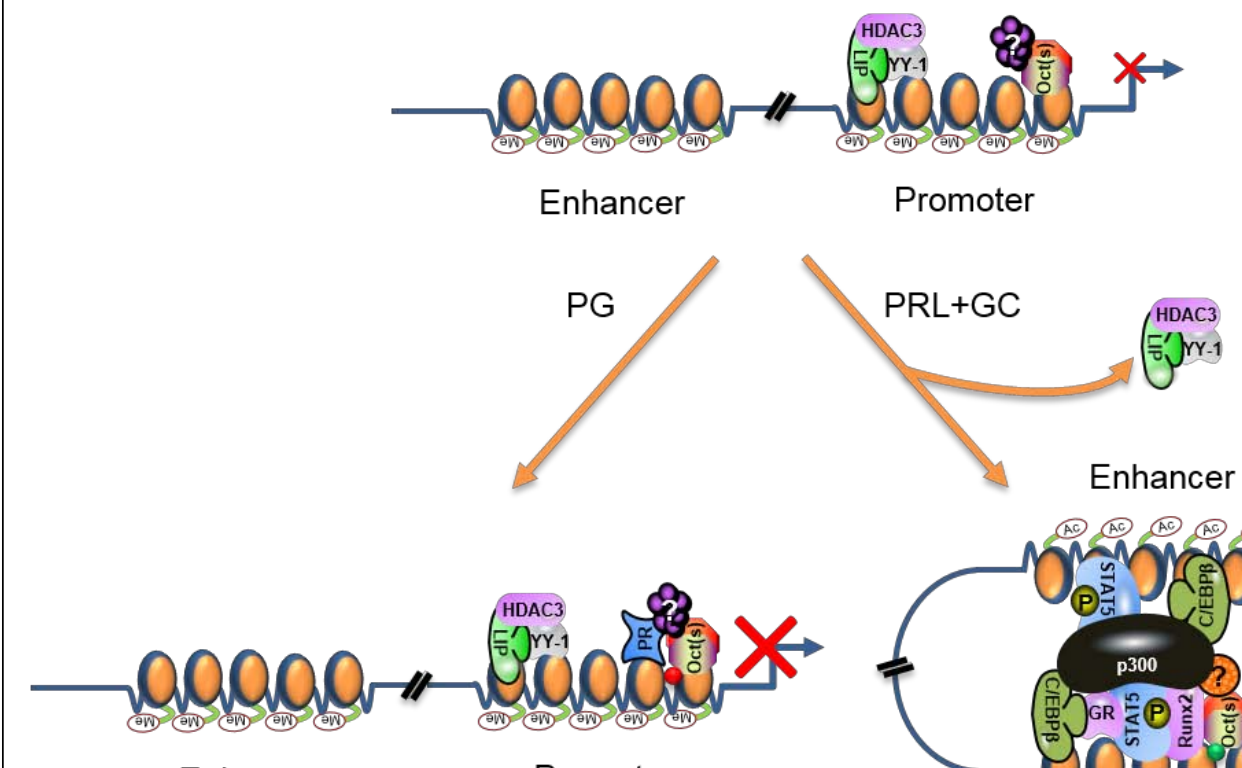

Enhancer

Promoter

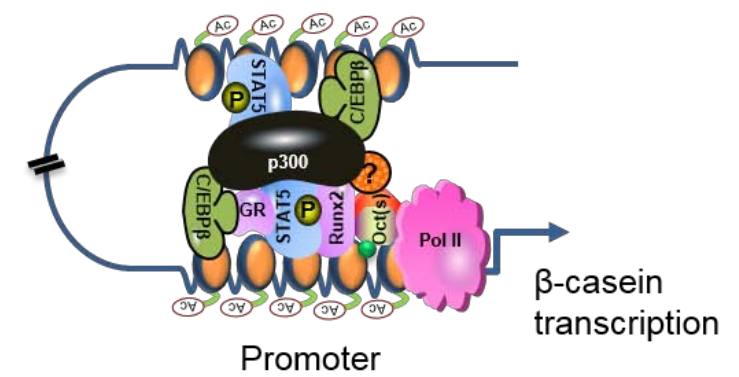


Fig. 3.

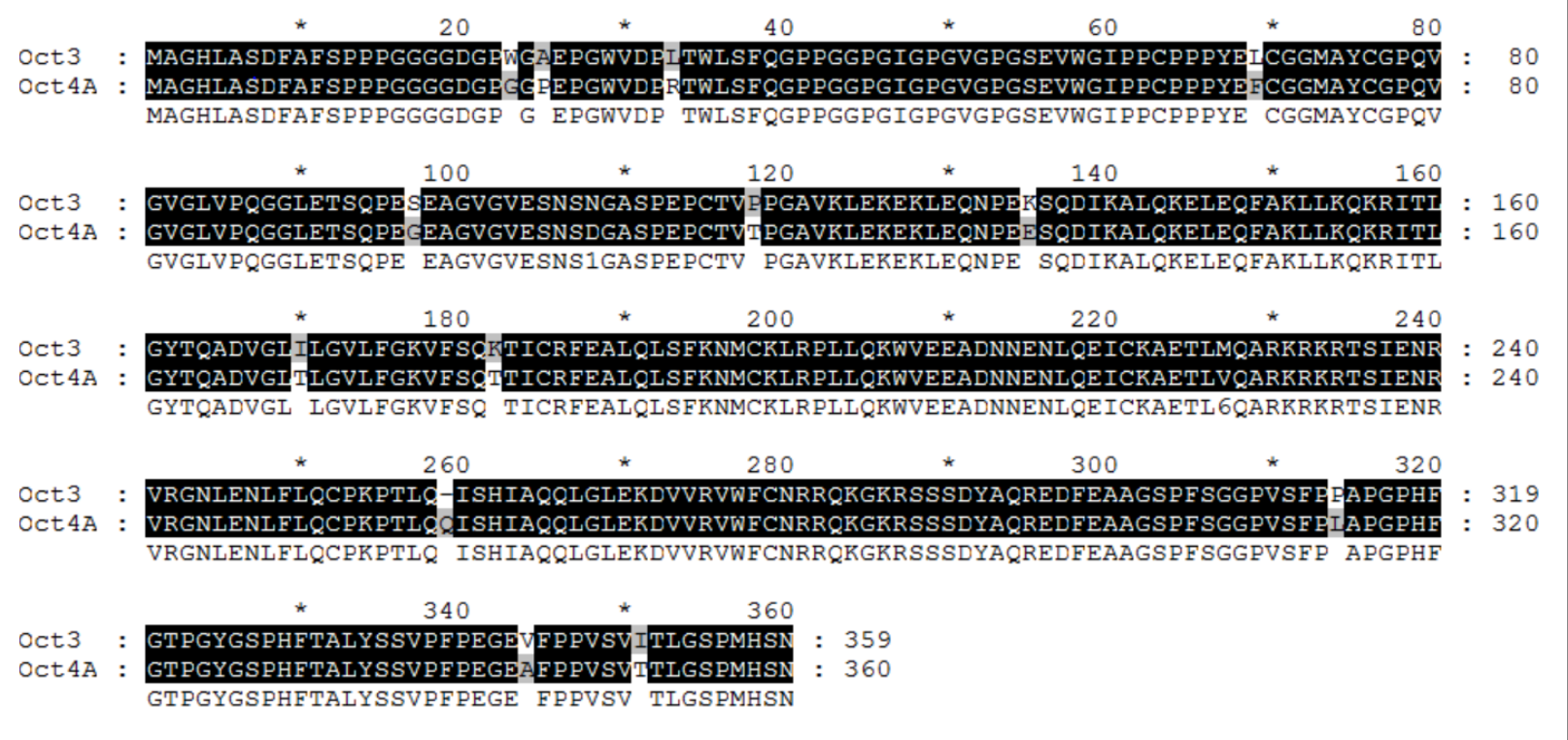




\section{References:}

[1] M.M. Babu, N.M. Luscombe, L. Aravind, M. Gerstein, S.A. Teichmann, Structure and evolution of transcriptional regulatory networks, Curr. Opin. Struct. Biol. 14 (2004) 283-291.

[2] A.H. Brivanlou, J.E. Darnell, Signal transduction and the control of gene expression, Science, 295 (2002) 813-818.

[3] H. Schöler, A.K. Hatzopoulos, R. Balling, N. Suzuki, P. Gruss, A family of octamer-specific proteins present during mouse embryogenesis: evidence for germline-specific expression of an Oct factor, EMBO J. 8 (1989) 2543-2550.

[4] F.-Q. Zhao, Y. Zheng, B. Dong, T. Oka, Cloning, genomic organization, expression, and effect on $\beta$ casein promoter activity of a novel isoform of the mouse Oct-1 transcription factor, Gene 326 (2004) 175-187.

[5] A.S. Goldsborough, L.E. Healy, N.G. Copeland, D.J. Gilbert, N.A. Jenkins, K.R. Willison, A. Ashworth, Cloning, chromosomal localization and expression pattern of the POU domain gene Oct-11, Nucleic Acids Res. 21 (1993) 127-134.

[6] H.R. Schöler, Octamania: the POU factors in murine development, Trends Genet. 7 (1991) 323-329.

[7] R.A. Sturm, G. Das, W. Herr, The ubiquitous octamer-binding protein Oct-1 contains a POU domain with a homeo box subdomain, Genes Dev. 2 (1988) 1582-1599.

[8] R.G. Clerc, L.M. Corcoran, J.H. LeBowitz, D. Baltimore, P.A. Sharp, The B-cell-specific Oct-2 protein contains POU box-and homeo box-type domains, Genes Dev. 2 (1988) 1570-1581.

[9] M.H. Rosner, M.A. Vigano, K. Ozato, P.M. Timmons, F. Poirie, P.W. Rigby, L.M. Staudt, A POU-domain transcription factor in early stem cells and germ cells of the mammalian embryo, Nature 345 (1990) 686-692.

[10] D. Meijer, A. Graus, R. Kraay, A. Langeveld, M.P. Mulder, G. Grosveld, The octamer binding factor Oct6: cDNA cloning and expression in early embryonic cells, Nucleic Acids Res. 18 (1990) 7357-7365.

[11] S. Atanasoski, S.S. Toldo, U. Malipiero, E. Schreiber, R. Fries, A. Fontana, Isolation of the human genomic brain-2/N-Oct 3 gene (POUF3) and assignment to chromosome 6q16, Genomics 26 (1995) 272-280.

[12] E. Schreiber, A. Tobler, U. Malipiero, W. Schaffner, A. Fontana, cDNA cloning of human N-Oct 3, a nervous-system specific POU domain transcription factor binding to the octamer DNA motif, Nucleic Acids Res. 21 (1993) 253-258.

[13] X. He, M.N. Treacy, D.M. Simmons, H.A. Ingraham, L.W. Swanson, M.G. Rosenfeld, Expression of a large family of POU-domain regulatory genes in mammalian brain development, Nature 340 (1989) 35-41.

[14] P. Douville, S. Atanasoski, A. Tobler, A. Fontana, M. Schwab, The brain-specific POU-box gene Brn4 is a sex-linked transcription factor located on the human and mouse $\mathrm{X}$ chromosomes, Mamm. Genome. 5 (1994) 180-182.

[15] A.K. Ryan, M.G. Rosenfeld, POU domain family values: flexibility, partnerships, and developmental codes, Genes Dev. 11 (1997) 1207-1225.

[16] F.-Q. Zhao, Octamer-binding transcription factors: genomics and functions, Front. Biosci. (Landmark Ed). 18 (2013) 1051-1071.

[17] C. Fletcher, N. Heintz, R.G. Roeder, Purification and characterization of OTF-1, a transcription factor regulating cell cycle expression of a human histone $\mathrm{H} 2 \mathrm{~b}$ gene, Cell 51 (1987) 773-781.

[18] J. Kang, M. Gemberling, M. Nakamura, F.G. Whitby, H. Handa, W.G. Fairbrother, D. Tantin, A general mechanism for transcription regulation by Oct1 and Oct4 in response to genotoxic and oxidative stress, Genes Dev. 23 (2009) 208-222.

[19] H. Saito, T. Oka, Hormonally regulated double-and single-stranded DNA-binding complexes involved in mouse-casein gene transcription, J. Biol. Chem. 271 (1996) 8911-8918. 
[20] F.-Q. Zhao, K. Adachi, T. Oka, Involvement of Oct-1 in transcriptional regulation of $\beta$-casein gene expression in mouse mammary gland, Biochim. Biophys. Acta. 1577 (2002) 27-37.

[21] T. Thum, J. Borlak, LOX-1 receptor blockade abrogates oxLDL-induced oxidative DNA damage and prevents activation of the transcriptional repressor Oct-1 in human coronary arterial endothelium, J. Biol. Chem. 283 (2008) 19456-19464.

[22] P. Wang, Q. Wang, J. Sun, J. Wu, H. Li, N. Zhang, Y. Huang, B. Su, R.-k. Li, L. Liu, POU homeodomain protein Oct-1 functions as a sensor for cyclic AMP, J. Biol. Chem. 284 (2009) 26456-26465.

[23] N.G. dela Paz, S. Simeonidis, C. Leo, D.W. Rose, T. Collins, Regulation of NF-kB-dependent gene expression by the POU domain transcription factor Oct-1, J. Biol. Chem. 282 (2007) 8424-8434.

[24] V. Sebastiano, M. Dalvai, L. Gentile, K. Schubart, J. Sutter, G.-M. Wu, N. Tapia, D. Esch, J.-Y. Ju, K. Hübner, Oct1 regulates trophoblast development during early mouse embryogenesis, Development 137 (2010) 3551-3560.

[25] P. Wang, T. Jin, Oct-1 functions as a sensor for metabolic and stress signals, Islets 2 (2010) 46-48.

[26] X. Qian, F.-Q. Zhao, Current major advances in the regulation of milk protein gene expression, Crit. Rev. Eukaryot. Gene Expr. 24 (2014) 357-378.

[27] A. Shakya, R. Cooksey, J.E. Cox, V. Wang, D.A. McClain, D. Tantin, Oct1 loss of function induces a coordinate metabolic shift that opposes tumorigenicity, Nat. Cell Biol. 11 (2009) 320-327.

[28] M. Ng, V. Lam, C. Tam, A. Chan, W.Y. So, R. Ma, B. Zee, M. Waye, W. Mak, C. Hu, Association of the POU class 2 homeobox 1 gene (POU2F1) with susceptibility to Type 2 diabetes in Chinese populations, Diabet. Med. 27 (2010) 1443-1449.

[29] H. Macias, L. Hinck, Mammary gland development, Wiley Interdiscip. Rev. Dev. Biol. 1 (2012) 533557.

[30] T. Jin, D.R. Branch, X. Zhang, S. Qi, B. Youngson, P.E. Goss, Examination of POU homeobox gene expression in human breast cancer cells, Int. J. Cancer. 81 (1999) 104-112.

[31] F. Hassiotou, A.R. Hepworth, A.S. Beltran, M.M. Mathews, A.M. Stuebe, P.E. Hartmann, L. Filgueira, P. Blancafort, Expression of the pluripotency transcription factor OCT4 in the normal and aberrant mammary gland, Front Oncol. 3 (2013) 79.

[32] B. Dong, F.-Q. Zhao, Expression of the Oct-2 transcription factor in mouse mammary gland and cloning and characterization of a novel Oct-2 isoform, Cell Tissue Res. 328 (2007) 595-606.

[33] J. Kang, A. Shakya, D. Tantin, Stem cells, stress, metabolism and cancer: a drama in two Octs, Trends Biochem. Sci. 34 (2009) 491-499.

[34] W. Herr, R. Sturm, R. Clerc, L. Corcoran, D. Baltimore, P. Sharp, H. Ingraham, M. Rosenfeld, M. Finney, G. Ruvkun, The POU domain: A large conserved region in the mammalian pit-1, oct-1, oct-2 and Caenorhabditis elegans unc-86 gene products, Genes Dev. 2 (1988) 1513-1516.

[35] J.D. Klemm, M.A. Rould, R. Aurora, W. Herr, C.O. Pabo, Crystal structure of the Oct-1 POU domain bound to an octamer site: DNA recognition with tethered DNA-binding modules, Cell 77 (1994) 2132.

[36] N. Dekker, M. Cox, R. Boelens, C.P. Verrijzer, P.C. van der Vliet, R. Kaptein, Solution structure of the POU-specific DNA-binding domain of Oct-1, Nature 362 (1993) 852-855.

[37] M.C. Botfield, A. Jancso, M.A. Weiss, Biochemical characterization of the Oct-2 POU domain with implications for bipartite DNA recognition, Biochemistry 31 (1992) 5841-5848.

[38] L. Nieto, G. Joseph, A. Stella, P. Henri, O. Burlet-Schiltz, B. Monsarrat, E. Clottes, M. Erard, Differential effects of phosphorylation on DNA binding properties of $\mathrm{N}$ Oct-3 are dictated by protein/DNA complex structures, J. Mol. Biol. 370 (2007) 687-700.

[39] D. Tantin, M. Gemberling, C. Callister, W. Fairbrother, High-throughput biochemical analysis of in vivo location data reveals novel distinct classes of POU5F1 (Oct4)/DNA complexes, Genome Res. 18 (2008) 631-639. 
[40] A. Reményi, A. Tomilin, E. Pohl, K. Lins, A. Philippsen, R. Reinbold, H.R. Schöler, M. Wilmanns, Differential dimer activities of the transcription factor Oct-1 by DNA-induced interface swapping, Mol. Cell. 8 (2001) 569-580.

[41] C.P. Verrijzer, A.J. Kal, P. Van der Vliet, The DNA binding domain (POU domain) of transcription factor oct-1 suffices for stimulation of DNA replication, EMBO J. 9 (1990) 1883-1888.

[42] I. Kemler, E. Bucher, K. Seipel, M.M. Müller-Immerglück, W. Schaffner, Promoters with the octamer DNA motif (ATGCAAAT) can be ubiquitous or cell type-specific depending on binding affinity of the octamer site and Oct-factor concentration, Nucleic Acids Res. 19 (1991) 237-242.

[43] M. Strubin, J.W. Newell, P. Matthias, OBF-1, a novel B cell-specific coactivator that stimulates immunoglobulin promoter activity through association with octamer-binding proteins, Cell 80 (1995) 497-506.

[44] V.E. Wang, T. Schmidt, J. Chen, P.A. Sharp, D. Tantin, Embryonic lethality, decreased erythropoiesis, and defective octamer-dependent promoter activation in Oct-1-deficient mice, Mol. Cell Biol. 24 (2004) 1022-1032.

[45] B. Andersen, M.D. Schonemann, S.E. Flynn, R.V. Pearse, H. Singh, M.G. Rosenfeld, Skn-1a and Skn-1i: two functionally distinct Oct-2-related factors expressed in epidermis, Science 260 (1993) 78-82.

[46] J. Hildesheim, U. Kuhn, C.L. Yee, R.A. Foster, K.B. Yancey, J.C. Vogel, The hSkn-1a POU transcription factor enhances epidermal stratification by promoting keratinocyte proliferation, J. Cell Sci. 114 (2001) 1913-1923.

[47] R.P. Friedrich, B. Schlierf, E.R. Tamm, M.R. Bösl, M. Wegner, The class III POU domain protein Brn-1 can fully replace the related Oct- 6 during schwann cell development and myelination, Mol. Cell Biol. 25 (2005) 1821-1829.

[48] M. Wolf, P. Lommes, E. Sock, S. Reiprich, R.P. Friedrich, J. Kriesch, C.C. Stolt, J.R. Bermingham, M. Wegner, Replacement of related POU transcription factors leads to severe defects in mouse forebrain development, Dev. Biol. 332 (2009) 418-428.

[49] M. Pesce, H.R. Schöler, Oct - 4: Control of totipotency and germline determination, Mol. Reprod. Dev. 55 (2000) 452-457.

[50] M.-H. Tai, C.-C. Chang, L.K. Olson, J.E. Trosko, Oct4 expression in adult human stem cells: evidence in support of the stem cell theory of carcinogenesis, Carcinogenesis 26 (2005) 495-502.

[51] D. Tantin, Oct transcription factors in development and stem cells: insights and mechanisms, Development, 140 (2013) 2857-2866.

[52] A. Shakya, J. Kang, J. Chumley, M.A. Williams, D. Tantin, Oct1 is a switchable, bipotential stabilizer of repressed and inducible transcriptional states, J Biol Chem. 286 (2011) 450-459.

[53] A. Shakya, C. Callister, A. Goren, N. Yosef, N. Garg, V. Khoddami, D. Nix, A. Regev, D. Tantin, Pluripotency transcription factor Oct4 mediates stepwise nucleosome demethylation and depletion, Mol. Cell Biol. 35 (2015) 1014-1025.

[54] X. Qian, F.-Q. Zhao, Interactions of the ubiquitous octamer-binding transcription factor-1 with both the signal transducer and activator of transcription 5 and the glucocorticoid receptor mediate prolactin and glucocorticoid-induced $\beta$-casein gene expression in mammary epithelial cells, Int. J. Biochem. Cell Biol. 45 (2013) 724-735.

[55] X. Qian, F.-Q. Zhao, Collaborative interaction of Oct-2 with Oct-1 in transactivation of lactogenic hormones-induced $\beta$-casein gene expression in mammary epithelial cells, Gen. Comp. Endocrinol. 204 (2014) 185-194.

[56] B. Dong, F.-Q. Zhao, Involvement of the ubiquitous Oct-1 transcription factor in hormonal induction of beta-casein gene expression, Biochem. J. 401 (2007) 57-64.

[57] B. Dong, C. Huang, D. Li, F.-Q. Zhao, Oct-1 functions as a transactivator in the hormonal induction of $\beta$-casein gene expression, Mol. Cell Biochem. 328 (2009) 93-99. 
[58] M. Rijnkels, D. Wheeler, H. De Boer, F. Pieper, Structure and expression of the mouse casein gene locus, Mamm. Genome 8 (1997) 9-15.

[59] R. Ball, R. Friis, C. Schoenenberger, W. Doppler, B. Groner, Prolactin regulation of beta-casein gene expression and of a cytosolic 120-kd protein in a cloned mouse mammary epithelial cell line, EMBO J. 7 (1988) 2089-2095.

[60] F. Hassiotou, A. Beltran, E. Chetwynd, A.M. Stuebe, A.J. Twigger, P. Metzger, N. Trengove, C.T. Lai, L. Filgueira, P. Blancafort, Breastmilk is a novel source of stem cells with multilineage differentiation potential, Stem Cells 30 (2012) 2164-2174.

[61] M. Rijnkels, L. Elnitski, W. Miller, J.M. Rosen, Multispecies comparative analysis of a mammalianspecific genomic domain encoding secretory proteins, Genomics, 82 (2003) 417-432.

[62] P. Winklehner-Jennewein, S. Geymayer, J. Lechner, T. Welte, L. Hansson, S. Geley, W. Doppler, A distal enhancer region in the human $\beta$-casein gene mediates the response to prolactin and glucocorticoid hormones, Gene 217 (1998) 127-139.

[63] J.M. Rosen, S.L. Wyszomierski, D. Hadsell, Regulation of milk protein gene expression, Annu. Rev. Nutr. 19 (1999) 407-436.

[64] E.B. Kabotyanski, M. Rijnkels, C. Freeman-Zadrowski, A.C. Buser, D.P. Edwards, J.M. Rosen, Lactogenic hormonal induction of long distance interactions between $\beta$-casein gene regulatory elements, J. Biol. Chem. 284 (2009) 22815-22824.

[65] M. Rijnkels, E. Kabotyanski, M.B. Montazer-Torbati, C.H. Beauvais, Y. Vassetzky, J.M. Rosen, E. Devinoy, The epigenetic landscape of mammary gland development and functional differentiation, J. Mammary Gland Biol. Neoplasia. 15 (2010) 85-100.

[66] M. Rijnkels, E. Kabotyanski, A. Shore, J.M. Rosen, The chromatin landscape of the casein gene locus, Horm. Mol. Biol. Clin. Investig. 10 (2012) 201-205.

[67] T. Bauknecht, R.H. See, Y. Shi, A novel C/EBP beta-YY1 complex controls the cell-type-specific activity of the human papillomavirus type 18 upstream regulatory region, J. Virol. 70 (1996) 76957705.

[68] V.S. Meier, B. Groner, The nuclear factor YY1 participates in repression of the beta-casein gene promoter in mammary epithelial cells and is counteracted by mammary gland factor during lactogenic hormone induction, Mol. Cell Biol. 14 (1994) 128-137.

[69] W.-M. Yang, Y.-L. Yao, J.-M. Sun, J.R. Davie, E. Seto, Isolation and characterization of cDNAs corresponding to an additional member of the human histone deacetylase gene family, J. Biol. Chem. 272 (1997) 28001-28007.

[70] E.B. Kabotyanski, M. Huetter, W. Xian, M. Rijnkels, J.M. Rosen, Integration of prolactin and glucocorticoid signaling at the $\beta$-casein promoter and enhancer by ordered recruitment of specific transcription factors and chromatin modifiers, Mol. Endocrinol. 20 (2006) 2355-2368.

[71] A.C. Buser, A.E. Obr, E.B. Kabotyanski, S.L. Grimm, J.M. Rosen, D.P. Edwards, Progesterone receptor directly inhibits $\beta$-casein gene transcription in mammary epithelial cells through promoting promoter and enhancer repressive chromatin modifications, Mol. Endocrinol. 25 (2011) 955-968.

[72] E. Pfitzner, R. Jahne, M. Wissler, E. Stoecklin, B. Groner, p300/CREB-binding protein enhances the prolactin-mediated transcriptional induction through direct interaction with the transactivation domain of Stat5, but does not participate in the Stat5-mediated suppression of the glucocorticoid response, Mol. Endocrinol. 12 (1998) 1582-1593.

[73] C.K. Inman, N. Li, P. Shore, Oct-1 counteracts autoinhibition of Runx2 DNA binding to form a novel Runx2/Oct-1 complex on the promoter of the mammary gland-specific gene $\beta$-casein, Mol. Cell Biol. 25 (2005) 3182-3193.

[74] A.C. Buser, E.K. Gass-Handel, S.L. Wyszomierski, W. Doppler, S.A. Leonhardt, J. Schaack, J.M. Rosen, H. Watkin, S.M. Anderson, D.P. Edwards, Progesterone receptor repression of prolactin/signal 
transducer and activator of transcription 5-mediated transcription of the $\beta$-casein gene in mammary epithelial cells, Mol. Endocrinol. 21 (2007) 106-125.

[75] G.G. Préfontaine, R. Walther, W. Giffin, M.E. Lemieux, L. Pope, R.J. Haché, Selective binding of steroid hormone receptors to octamer transcription factors determines transcriptional synergism at the mouse mammary tumor virus promoter, J. Biol. Chem. 274 (1999) 26713-26719.

[76] C. Åstrand, S. Belikov, Ö. Wrange, Histone acetylation characterizes chromatin presetting by NF1 and Oct1 and enhances glucocorticoid receptor binding to the MMTV promoter, Exp. Cell Res. 315 (2009) 2604-2615.

[77] B.E. Bernstein, T.S. Mikkelsen, X. Xie, M. Kamal, D.J. Huebert, J. Cuff, B. Fry, A. Meissner, M. Wernig, K. Plath, A bivalent chromatin structure marks key developmental genes in embryonic stem cells, Cell 125 (2006) 315-326.

[78] X. Chen, H. Xu, P. Yuan, F. Fang, M. Huss, V.B. Vega, E. Wong, Y.L. Orlov, W. Zhang, J. Jiang, Integration of external signaling pathways with the core transcriptional network in embryonic stem cells, Cell 133 (2008) 1106-1117.

[79] L.-S. Yeap, K. Hayashi, M.A. Surani, ERG-associated protein with SET domain (ESET)-Oct4 interaction regulates pluripotency and represses the trophectoderm lineage, Epigenetics Chromatin 2 (2009) 12.

[80] P. Yuan, J. Han, G. Guo, Y.L. Orlov, M. Huss, Y.-H. Loh, L.-P. Yaw, P. Robson, B. Lim, H.-H. Ng, Eset partners with Oct4 to restrict extraembryonic trophoblast lineage potential in embryonic stem cells, Genes Dev. 23 (2009) 2507-2520.

[81] A. Whitmarsh, R. Davis, Regulation of transcription factor function by phosphorylation, Cell Mol. Life Sci. 57 (2000) 1172-1183.

[82] C.I. Holmberg, S.E. Tran, J.E. Eriksson, L. Sistonen, Multisite phosphorylation provides sophisticated regulation of transcription factors, Trends Biochem. Sci. 27 (2002) 619-627.

[83] V.E. Wang, D. Tantin, J. Chen, P.A. Sharp, B cell development and immunoglobulin transcription in Oct-1-deficient mice, Proc. Natl. Acad. Sci. U. S. A. 101 (2004) 2005-2010.

[84] C.P. Verrijzer, J. van Oosterhout, P.C. van der Vliet, The Oct-1 POU domain mediates interactions between Oct-1 and other POU proteins, Mol. Cell Biol. 12 (1992) 542-551.

[85] C.J. Watson, W.T. Khaled, Mammary development in the embryo and adult: a journey of morphogenesis and commitment, Development 135 (2008) 995-1003.

[86] R. Kumar, R. Vadlamudi, L. Adam, Apoptosis in mammary gland and cancer, Endocr. Relat. Cancer 7 (2000) 257-269.

[87] A. Marti, H. Lazar, P. Ritter, R. Jaggi, Transcription factor activities and gene expression during mouse mammary gland involution, J. Mammary Gland Biol. Neoplasia. 4 (1999) 145-152.

[88] A. Marti, B. Jehn, E. Costello, N. Keon, G. Ke, F. Martin, R. Jaggi, Protein kinase A and AP-1 (cFos/JunD) are induced during apoptosis of mouse mammary epithelial cells, Oncogene 9 (1994) 1213-1223.

[89] R. Jaggi, A. Marti, K. Guo, Z. Feng, R.R. Friis, Regulation of a physiological apoptosis: mouse mammary involution, J. Dairy Sci. 79 (1996) 1074-1084.

[90] T.C. Chen, D.R. Hinton, R. Zidovetzki, F.M. Hofman, Up-regulation of the cAMP/PKA pathway inhibits proliferation, induces differentiation, and leads to apoptosis in malignant gliomas, Lab. Invest. 78 (1998) 165-174.

[91] Y. Shi, C. Desponts, J.T. Do, H.S. Hahm, H.R. Schöler, S. Ding, Induction of pluripotent stem cells from mouse embryonic fibroblasts by Oct4 and Klf4 with small-molecule compounds, Cell Stem Cell 3 (2008) 568-574.

[92] S. Roy, P. Gascard, N. Dumont, J. Zhao, D. Pan, S. Petrie, M. Margeta, T.D. Tlsty, Rare somatic cells from human breast tissue exhibit extensive lineage plasticity, Proc. Natl. Acad. Sci. U. S. A. 110 (2013) 4598-4603. 
[93] P. Wang, D. Branch, M. Bali, G. Schultz, P. Goss, T. Jin, The POU homeodomain protein OCT3 as a potential transcriptional activator for fibroblast growth factor-4 (FGF-4) in human breast cancer cells, Biochem. J. 375 (2003) 199-205.

[94] A.S. Beltran, A.G. Rivenbark, B.T. Richardson, X. Yuan, H. Quian, J.P. Hunt, E. Zimmerman, L.M. Graves, P. Blancafort, Generation of tumor-initiating cells by exogenous delivery of OCT4 transcription factor, Breast Cancer Res. 13 (2011) R94.

[95] S.W. McLeskey, J. Kurebayashi, S.F. Honig, J. Zwiebel, M.E. Lippman, R.B. Dickson, F.G. Kern, Fibroblast growth factor 4 transfection of MCF-7 cells produces cell lines that are tumorigenic and metastatic in ovariectomized or tamoxifen-treated athymic nude mice, Cancer Res. 53 (1993) 21682177.

[96] D. Wang, P. Lu, H. Zhang, M. Luo, X. Zhang, X. Wei, J. Gao, Z. Zhao, C. Liu, Oct-4 and nanog promote the epithelial-mesenchymal transition of breast cancer stem cells and are associated with poor prognosis in breast cancer patients, Oncotarget 5 (2014) 10803-10815.

[97] G. Cotsarelis, T.-T. Sun, R.M. Lavker, Label-retaining cells reside in the bulge area of pilosebaceous unit: implications for follicular stem cells, hair cycle, and skin carcinogenesis, Cell 61 (1990) 13291337.

[98] T. Hu, S. Liu, D.R. Breiter, F. Wang, Y. Tang, S. Sun, Octamer 4 small interfering RNA results in cancer stem cell-like cell apoptosis, Cancer Res. 68 (2008) 6533-6540.

[99] D. Ponti, A. Costa, N. Zaffaroni, G. Pratesi, G. Petrangolini, D. Coradini, S. Pilotti, M.A. Pierotti, M.G. Daidone, Isolation and in vitro propagation of tumorigenic breast cancer cells with stem/progenitor cell properties, Cancer Res. 65 (2005) 5506-5511.

[100] D. Pain, G.-W. Chirn, C. Strassel, D.M. Kemp, Multiple retropseudogenes from pluripotent cellspecific gene expression indicates a potential signature for novel gene identification, J.Biol. Chem. 280 (2005) 6265-6268.

[101] G. Suo, J. Han, X. Wang, J. Zhang, Y. Zhao, Y. Zhao, J. Dai, Oct4 pseudogenes are transcribed in cancers, Biochem. Biophys. Res. Commun. 337 (2005) 1047-1051.

[102] J. Takeda, S. Seino, G.I. Bell, Human Oct3 gene family: cDNA sequences, alternative splicing, gene organization, chromosomal location, and expression at low levels in adult tissues, Nucleic Acids Res. 20 (1992) 4613-4620.

[103] J. Maddox, A. Shakya, S. South, D. Shelton, J.N. Andersen, S. Chidester, J. Kang, K.M. Gligorich, D.A. Jones, G.J. Spangrude, Transcription factor Oct1 is a somatic and cancer stem cell determinant, PLoS Genet. 8 (2012) e1003048. 